\title{
LA COHERENCIA IDEOLÓGICO-PROGRAMÁTICA DE LOS PARTIDOS POLÍTICOS EN CHILE Y MÉXICO: ¿EN QUÉ MEDIDA Y POR QUÉ LAS UBICACIONES DE LOS PARLAMENTARIOS COINCIDEN CON EL CONTENIDO DE LOS PROGRAMAS ELECTORALES?*
}

The Ideological-Programmatic Coherence of the Political Parties in Chile and Mexico: To What Extent and Why Do the Ideological Position of the Parliamentary Members Match with the Content of the Electoral Programs?

\section{ALDO ADRIÁN MARTÍNEZ-HERNÁNDEZ}

Universidad Autónoma de Aguascalientes, México

\section{NICOLÁS MIRANDA OLIVARES}

Universidad de Salamanca, España

\begin{abstract}
RESUMEN
La investigación desarrolla una propuesta metodológica para el estudio de la coherencia ideológico-programática de los partidos políticos. El texto diseña herramientas para el análisis espacial de distancia entre la ideología formal del partido, expresada por el programa electoral, y las posiciones ideológicas que los representantes legislativos otorgan a sus organizaciones partidistas. Para ello, se analizan los partidos de Chile y México, con la intención de determinar las diferencias entre los partidos políticos y las coaliciones en distintos sistemas. La investigación concluye que las coaliciones y partidos ubicados ideológicamente a la derecha de la escala, son menos coherentes que los ubicados a la izquierda, lo que es más evidente en contextos de gobiernos de coalición.
\end{abstract}

Palabras clave: ideología, programa electoral, coherencia partidista, índice de coherencia ideológico-programática

\begin{abstract}
This research develops a method for the study of the ideological-programmatic coherence of political parties. It designs tools for the spatial analysis of the distance between the formal ideology of a party - as determined by the content of its electoral platform - and the ideological positions that its legislative representatives attribute to their own party organization. To do this, we analyze political parties in Chile and Mexico, with the purpose of determining the differences between parties and coalitions in different systems. The research concludes that coalitions and parties located on the right of the political spectrum are less coherent than those located on the left - a feature that is most evident in the context of coalition governments.
\end{abstract}

Keywords: ideology, electoral program, party coherence, ideological-programmatic coherence index 


\section{INTRODUCCIÓN}

La estructuración programática de la competencia en las democracias representativas ha recorrido un largo proceso de institucionalización. Dicho recorrido ha prevalecido en contextos cuyos procesos democráticos se han consolidado (Budge et al. 2001; Budge 2006; Franzmann y Kaiser 2006; Freidenberg et al. 2006; Volkens 2007; Volkens et al. 2013; Volkens y Bara 2013). Sin embargo, el debate académico sobre los vínculos programáticos y el papel de la ideología en contextos democráticos de la tercera ola no ha despertado el mismo interés académico. La escasa producción académica sobre el estado que guarda la ideología en la definición de la competencia en América Latina ha sido obviada por los elementos personalistas de la política en la región, restando importancia a los efectos de la estructura ideológica de los partidos y coaliciones en los sistemas con democracias presidencialistas (Mainwaring y Scully 1995; Coppedge 1997, 1998; Alcántara 2004; Luna y Zechmeister 2005; Mainwaring y Torcal 2005; Kitschelt et al. 2010; Ruiz y Otero 2013; Volkens y Bara 2013; Luna 2014; Torcal 2015; Ares y Volkens 2017: 124).

En la breve literatura asociada yacen los estudios sobre la identificación de un eje programático como elemento de diferenciación ideológica de los partidos en América Latina (Ares y Volkens 2017). En ella se identifican al menos tres dimensiones analíticas. La primera asume, bajo la teoría de la representación, que los partidos políticos y ciudadanos manifiestan vínculos, los cuales se expresan en el grado de coincidencia en diferentes temas políticos: la congruencia programática. La segunda asume, bajo el análisis de las organizaciones partidistas, una postura que observa el nivel de unidad ideológica al interior de los partidos según sus miembros: la coherencia ideológica (Kitschelt et al. 2010; Ruiz y Otero 2013; Otero y Rodríguez 2014). La tercera asume que los sistemas de partidos se estructuran en ejes programáticos que determinan la competencia, lo cual indica que los partidos poseen (en mayor o menor grado) una identidad ideológica expresada en los programas electorales (Ares y Volkens 2017: 124).

A pesar de su relevancia teórica para el estudio de la representación y la propia teoría de partidos, estas tres visiones no han avanzado hacia el entendimiento de las dinámicas específicas de estructuración de la competencia y las posibles implicaciones en el diseño de los sistemas políticos. Por ello, el interés de esta investigación es aportar a la literatura especializada a partir del análisis de una condición previa a los efectos de la ideología en la estructuración de la competencia: la coherencia ideológica y programática de los partidos políticos. Lo anterior tiene que ver con una perspectiva relativamente nueva en el estudio de las organizaciones partidistas, que presta atención a la importancia de las dinámicas ideológicas en los procesos de representación democrática (Ruiz y Otero 2013; Otero y Rodríguez 2014, 2015).

De acuerdo con lo anterior, la investigación realiza una propuesta metodológica para el estudio de la coherencia ideológico-programática de los partidos políticos, sustentada en el análisis espacial de la ideología formal de los 
partidos expresada en los programas electorales (cara externa) y la ideología que los propios representantes otorgan a sus organizaciones partidistas (cara interna). Esta consideración manifiesta la discusión de dos perspectivas teóricas que, a su vez, obligan a una delimitación metodológica que permita la comparación. La relevancia metodológica de esta aproximación no solo atiende una veta de investigación poco desarrollada, también discute la pertinencia ideológica en procesos de consolidación democrática. En ese sentido, el texto desarrolla mecanismos para el análisis de la coherencia ideológica a partir de dos preguntas que guían la investigación: ¿son los miembros de los partidos coherentes ideológicamente respecto a los programas electorales presentados por sus partidos y, en su caso, coaliciones? y ¿existe un patrón ideológico que determine el nivel de coherencia?

Para dar respuesta a los cuestionamientos anteriores, el artículo se estructura en cuatro secciones. La primera desarrolla los principales lineamientos teóricos de la investigación, puntualizando la importancia de la coherencia como elemento de consideración metodológica para el estudio de la ideología de los partidos políticos, en específico, en el contexto latinoamericano. La segunda sección desarrolla la propuesta metodológica, destacando la creación del índice de coherencia ideológico-programática, el cual permite la comparación en diversos contextos. Para ello, la investigación hace uso de dos bases de datos que se relacionan directamente con las dos perspectivas teóricas. La primera es el Party Manifesto Project (MARPOR), de la cual se obtiene la identificación programática de los partidos políticos de acuerdo con sus plataformas electorales. La segunda es el Base de Datos de Élites Latinoamericanas-Universidad de Salamanca (PELA-USAL), de la que se obtienen las posiciones ideológicas de los partidos según sus representantes en el Congreso. La tercera sección avanza hacia la observación empírica de los indicadores propuestos en la realidad partidista latinoamericana, puntualizando las discrepancias de la coherencia ideológica tanto de los partidos como de las coaliciones en dos sistemas de partidos: México y Chile. Finalmente, el cuarto apartado cierra con las conclusiones.

\section{EL MODELO DE PARTIDO RESPONSABLE: FUNDAMENTOS TEÓRICOS DE LA COHERENCIA}

Los cambios ocurridos a nivel político con la ampliación del derecho a sufragio, la regularidad de las elecciones, así como el surgimiento de los partidos de masas, evidenciaron un impacto importante en el desarrollo de la teoría y práctica de la representación política (Manin 1998: 237-241). Lo anterior representó una tensión en la controversia mandato-independencia, argumentando que, en la medida en que todos participaran en la elección de los representantes, estos últimos tendrían menos autonomía para actuar y quedarían expuestos al control de los electores (responsiveness y accountability frente a los electores) (Avendaño 2008: 102-103). Esta dicotomía no solo evidenció la necesidad de incorporar nuevos modelos teóricos para el estudio de la representación, sino 
que ubicó a los partidos políticos en el centro del análisis. Dentro de este nuevo esquema de representación, los partidos han sido los encargados de recoger los problemas de la ciudadanía y formular soluciones que serán ofrecidas durante los comicios. Con ello, los electores tienen la posibilidad de decidir entre las alternativas existentes, con la promesa de ser implementadas una vez ganados los cargos por los cuales se compite (Dalton 1985: 268-271; Budge y Hofferbert 1990: 112; Manin et al. 2002: 19-20; McDonald y Budge 2005: 3). En este marco, surge un nuevo modelo de partido: el responsible party model (Dalton 1985: 269-270). Este modelo presenta una serie de principios que tienen que ver con procesos electorales más competitivos para el acceso al poder, con la oferta de programas y propuestas electorales distintas para que los electores puedan elegir, y con el flujo de información suficiente para que los electores puedan recompensar o castigar a los partidos, en las elecciones, de acuerdo con su actuar en la legislatura anterior. El modelo de partido responsable exige que se cumpla una serie de supuestos (Dalton 1985: 270; Thomassen y Schmitt 1997: 168):

"1. Voters have a choice of parties in competitive elections, i.e., they can choose between at least two parties with different programmes. 2. The internal cohesion, or party discipline, of political parties is sufficient to enable them to implement their policy programme. 3. Voters have policy preferences. 4. Voters are aware of the differences between the programmes of different political parties. 5. Electors vote according to their policy preferences, i.e., they choose the party that best represents their policy preferences" (Thomassen y Schmitt 1997: 168).

Con los principios anteriores, el modelo entiende que la representación tiene relación con una característica propia de la emisión del voto. Es decir, el votante ejerce su derecho a elegir a los representantes considerando la oferta en sus programas electorales, lo cual tiene que ver con la estructuración de la competencia en torno a las teorías del voto ideológico y programático (Dalton 1985; Budge y Hofferbert 1990; Manin et al. 2002; McDonald y Budge 2005; Volkens y Bara 2013; Otero y Rodríguez 2014, 2015; Ares y Volkens 2017).

Desde esta perspectiva, la representación cimentada en vínculos programáticos entre representantes y representados es uno de los elementos centrales del modelo de partido responsable, debido a que, por un lado, establece la relevancia de los programas electorales de los partidos políticos en la estructuración de la competencia y, por el otro, identifica la importancia del cumplimiento de las directrices ofertadas por los partidos al llegar al poder. En este sentido, los vínculos programáticos funcionarán mejor en la medida en que exista congruencia entre representantes, representados y las políticas que se ejecuten (Dalton 1985). La existencia de vínculos programáticos permite, además, que el tipo de accountability que realiza la ciudadanía en las elecciones se base en la capacidad de los partidos para cumplir con sus programas electorales, evaluando la gestión realizada por los representantes en ejercicio (Morales 2014: 66). De ahí que, si se han desviado del mandato o de las promesas por ellos realizadas, o no han actuado acorde a sus intereses o el interés general, pueden ser castigados por los electores. Según este modelo, los partidos deben 
actuar unidos y votar en bloque en la legislatura de acuerdo con las directrices del partido (disciplina de partido) (Miller y Stokes 1963; Dalton 1985: 270-271; Manin et al. 2002: 20 y 32-37; Samuels y Shugart 2006: 4-5).

El otro elemento clave del modelo de partido responsable son los propios programas electorales. Estos son vistos como el texto donde se "establecen las condiciones funcionales esenciales para el éxito de la representación política" (Ares y Miranda 2017: 2). La importancia de los programas viene dada por cuanto son un texto oficial que representa las posiciones de los actores políticos (partidos y representantes) en cada elección, donde se ofrece a la ciudadanía modelos y ofertas políticas, teóricamente distintas, con el fin de ganar las elecciones (Alonso et al. 2012; Alcántara y Cabezas 2013; D'Alessandro 2013). Los programas específicamente proveen información sobre las estrategias electorales de los partidos, además de ser un medio para la agregación de intereses, lo cual salvaguarda que los intereses del partido estén por sobre los intereses particulares (Alcántara y Cabezas 2013: 29). En esta línea académica, el proceso de elaboración de programas electorales adquiere relevancia teórica debido a que permite definir el grado de discreción de la dirigencia de los partidos en torno a sus posicionamientos ideológicos (Alcántara y Cabezas 2013: 29).

Una de las críticas hacia los sistemas de partidos latinoamericanos y, con ello, la dificultad del viaje teórico conceptual del partido responsable es que los partidos tienen vínculos programáticos débiles o inexistentes entre sus miembros y con la ciudadanía, pues la relación estaría mediada por el personalismo y el clientelismo. Es más, existen partidos que estarían próximos a ser maquinarias electorales más que instituciones ideológicas (Alcántara 2004). Los partidos políticos latinoamericanos presentan una serie de problemas que los alejan de los ideales teóricos. Sin embargo, tienen un actuar y un papel en la sociedad similares a los partidos occidentales, que van desde la conformación de sus principios programáticos hasta la definición de sus estructuras organizativas (Alcántara 2004: 156).

\section{COHERENCIA IDEOLÓGICA INTRAPARTIDISTA}

Uno de los principios de la teoría de partido responsable es la existencia de cohesión intrapartidista —internal cohesion - que les permita implementar su programa electoral. Los trabajos precursores realizaron aproximaciones desde la congruencia entre representantes y representados. Tomando como referencia la controversia mandato-independencia, también las ideas desarrolladas por Fenichel Pitkin (2013) sobre representación descriptiva y sustantiva, junto al rol de los partidos políticos, los primeros trabajos analizaron si existía cercanía, o no, entre las posturas de las personas y los representantes y sus acciones en las asambleas, inicialmente en Estados Unidos (Miller y Stokes 1963), luego en Europa (Dalton 1985; Thomassen 1991, 1994) y también en América Latina (Luna y Zechmeister 2005; González y Queirolo 2013; Joignant et al. 2017). 
Sin embargo, el estudio de la unidad al interior del partido, tanto ideológica como programática, no manifestó la misma relevancia dentro de la teoría del partido responsable. Por ello surgen los estudios sobre la coherencia partidista, los cuales analizan el grado de acuerdo que existe en las preferencias y actitudes de los miembros de un partido hacia determinados objetos políticos (Ruiz y García 2003; Ruiz y Otero 2013). Como señala Ruiz (2006: 90), la coherencia partidista constituye una herramienta analítica que permite determinar "la fuerza de temas sustantivos en la competencia partidista". En este sentido, el grado de acuerdo en las posiciones de los miembros frente a diferentes temas revelará "si el partido está programáticamente preocupado y orientado hacia ellas" (Ruiz 2006: 90).

En términos metodológicos, la coherencia se puede dividir en dos dimensiones: ideológica y programática (Ruiz 2006: 91-92; 2007; Ruiz y Otero 2013: 41-43). La primera identifica cuán similar es la ubicación ideológica de un partido en términos agregados o en la autoubicación de sus miembros, lo cual permite comprender en qué medida se comparte, o no, un mismo proyecto político (Ruiz y Otero 2013: 41). En este ámbito, la ideología sirve como un atajo que resume las posiciones que el partido y sus miembros adoptan en diversos temas (Alcántara 2004). La coherencia programática, por otro lado, tiene como objetivo "medir las similitudes intra-partidistas en las posiciones de los miembros de un partido político en temas y políticas específicas" (Ruiz y Otero 2013: 42). Dependiendo del tema, podrá existir mayor o menor grado de coherencia dentro de un partido. A ello, Ruiz (2006) señala que estas posturas suelen ser de conocimiento público por medio de programas de partidos o discursos de sus líderes. Agrega que la coherencia programática "presenta vínculos teóricos y empíricos con la dimensión ideológica, constituyendo, en cierto sentido, una concreción de esta" (Ruiz 2006: 92) ${ }^{1}$.

\section{COHERENCIA IDEOLÓGICO-PROGRAMÁTICA: ENTRE PROGRAMAS ELECTORALES Y LOS REPRESENTANTES LEGISLATIVOS COMO PROPUESTA DE INVESTIGACIÓN}

A pesar de que la literatura proporciona distintas alternativas para el estudio de la cohesión interna, como la coherencia, la cristalización programática o la disciplina interna (Ruiz y García 2003: 74), dentro de esta delimitación teórica no existe una aproximación que permita definir que tanto los partidos, sus miembros y sus programas electorales coinciden, lo cual manifiesta no solo una carencia metodológica, sino una laguna teórica que no permite identificar

\footnotetext{
El índice de coherencia ideológica, según Ruiz y Otero (2013), evidencia qué tan unidos ideológicamente son los partidos políticos. El índice mide de 0 a n, indicando que, entre más alejado del 0 , más incoherencia ideológica. $\overline{x_{i}}$ : Promedio del partido, $x_{i}$ : Posiciones ideológicas de cada individuo, N: Número de entrevistados por partido$$
\mathrm{S}=\frac{\sqrt{\sum_{i=1}^{n}\left(x_{i}-\overline{x_{j}}\right)^{2}}}{n}
$$ 
sus efectos en los procesos de representación democrática. Por ello, surge la necesidad de identificar el grado en el que los miembros de los partidos y sus programas concuerdan. En este sentido, el concepto coherencia ideológicoprogramática infiere un análisis de las dinámicas ideológicas internas y externas de los partidos políticos y, a su vez, promueve un acercamiento metodológico al entramado ideológico de la representación. Este nuevo índice propuesto mantiene el concepto de coherencia, debido a que hace referencia al grado de acuerdo o cercanía entre los miembros del partido respecto a un determinado proyecto. Sin embargo, a diferencia de la coherencia, que utiliza solo las percepciones de los representantes legislativos, el nuevo índice compara esta visión con el posicionamiento ideológico de los partidos políticos construido a partir de sus propuestas programáticas expresadas en los programas electorales.

En concreto, la coherencia ideológico-programática reviste especial importancia a la identificación ideológica de los partidos en contextos de consolidación y estructuración de la competencia, así como de las propias dinámicas de la representación. Bajo este marco, la investigación define a la coherencia ideológico-programática como el grado de consenso ideológico entre el partido como aglutinador de percepciones de sus actores constitutivos (representantes) y el programa electoral emitido como la articulación formal de dicho consenso. En términos teóricos, un partido que es coherente tendría mayores elementos programáticos e ideológicos para la creación de vínculos más estables con sus representados. En este sentido, los partidos políticos que manifiestan niveles altos de coherencia poseen una claridad objetiva y compartida del programa político de la organización, con ello podrían actuar conforme a los lineamientos programáticos partidistas y, en consecuencia, manifestarían una representación política de mejor calidad. Esto último debido a que, en términos organizativos, el elector podría discernir entre organizaciones partidistas que interiorizan las plataformas electorales y partidos que no manifiestan una estructura homogénea de las políticas que promueven en los procesos electorales. En términos específicos, la coherencia en los procesos de representación es una herramienta empírica del responsiveness y del accountability, debido a que evidencia los niveles de dispersión de las promesas de campaña y sus efectos en los procesos de implementación subjetiva de la representación ejercida por los miembros de los partidos políticos. 
En términos operativos, la coherencia ideológica es la distancia entre la ubicación ideológica de los partidos políticos, según sus representantes, y la ideología del partido, según su programa electoral. Lo anterior proporciona una propuesta de construcción conceptual y teórica más sustantiva sobre la representación política y se manifiesta a partir de la estructuración ideológico-programática de los partidos políticos. Con base en esta consideración metodológica, se entiende que un partido es más coherente cuanto más cercana sea la posición ideológica del partido y sus miembros $\left(\operatorname{Im}_{\mathrm{a}}\right.$ ) con el programa electoral (Ipe $\mathrm{a}_{\mathrm{a}}$, como lo muestra el partido A en la figura 1. Esta concepción teórica pretende evidenciar qué tan coherentes son los partidos respecto a las propuestas enmarcadas en el programa. De acuerdo con lo anterior, la coherencia es observada en términos de distancia, entendiendo que un partido que manifiesta niveles altos de coherencia es aquel que tiene menor distancia entre su programa electoral y la posición ideológica media de sus miembros (Figura 1).

Figura 1. Ejemplo teórico de la coherencia ideológico-programática

\begin{tabular}{|c|c|c|c|c|c|c|c|c|c|c|}
\hline $\begin{array}{l}\text { Ubicación } \\
\text { ideológica }\end{array}$ & $\begin{array}{c}1 \\
\text { Izquierda }\end{array}$ & 2 & 3 & 4 & 5 & 6 & 7 & 8 & 9 & $\begin{array}{c}10 \\
\text { Derecha }\end{array}$ \\
\hline Partido A & & & & & $\begin{array}{l}\mathrm{Im}_{\mathrm{a}} \\
\text { I---- } \\
\text { Dist }\end{array}$ & $\begin{array}{l}\text { Ipe }_{\text {a }} \\
\text { ia } 1 \\
\text { ia }\end{array}$ & & & & \\
\hline Partido B & $\begin{array}{c}\mathrm{Im}_{\mathrm{b}} \\
\mathrm{I}\end{array}$ & & & & Dist & ia 2 & . & & & Ipe $_{b}$ \\
\hline
\end{tabular}

Fuente: elaboración propia

\section{METODOLOGÍA}

Esta investigación utiliza una base de datos comparativa que contiene información de dos proyectos de investigación: Party Manifesto Project (Manifesto Research on Political Representation: MARPOR) (Volkens et al. 2016) y el Proyecto de Élites Latinoamericanas-Universidad de Salamanca (PELA-USAL) (Alcántara 2018). Ambos proyectos tienen en común el estudio de la representación: el primero a través de las posiciones y énfasis que los partidos tienen frente a determinados temas a través de los programas electorales, mientras que el segundo busca comprender las opiniones y actitudes de los representantes de los partidos políticos en la Cámara baja. Las dos perspectivas están relacionadas entre sí. Tal como se enunció, la teoría del partido responsable tiene entre sus 
principios la unidad del partido y la necesidad de compartir entre ellos y sus electores una visión común acerca de los problemas y soluciones políticas, económicas y sociales. De esta manera, se podría esperar que los partidos más institucionalizados compartan posiciones similares entre sus programas electorales y los representantes legislativos. La primera base de datos es MARPOR, la cual ofrece medidas estandarizadas sobre los programas electorales de los partidos políticos. El análisis de contenido en cuasifrases de los programas electorales elaborado por MARPOR, proporciona la posibilidad de medir la ideología en dos binomios: la escala izquierda-derecha RILE (Laver y Budge 1992; Budge 2006, 2013). La escala RILE está constituida por veintiséis categorías, según lo establecido por Laver y Budge (1992) y Budge (2013) y adaptada para el contexto latinoamericano (Ares y Volkens 2017: 126)2

\section{Dimensión Derecha-Izquierda (RILE-MARPOR)}

RILE $=$ Derecha (per104 Military: Positive + per201 Freedom and Human Rights + per203 Constitutionalism: Positive + per305 Political Authority + per401 Free Market Economy + per402 Economic Incentives + per407 Protectionism: Negative + per414 Economic Orthodoxy + per505 Welfare State Limitation + per601 National Way of Life: Positive + per603 Traditional Morality: Positive + per605 Law and Order + per606 Civic Mindedness: Positive) - Izquierda (per103 Anti-imperialism + per105 Military: Negative + per106 Peace + per107 Internationalism: Positive + per202 Democracy + per403 Market Regulation + per404 Economic Planning + per406 Protectionism: Positive + per412 Controlled Economy + per413 Nationalization + per504 Welfare State Expansion + per506 Education Expansion + per701 Labour Groups: Positive).

La escala original, que va de -100 a 100 (siendo -100 extrema izquierda y 100 extrema derecha), proporcionada por el MARPOR, se transforma a la escala de uno a diez, en donde uno es izquierda y diez es derecha. Lo anterior se realiza con la finalidad de comparación de la escala RILE-MARPOR y la escala del LR PELA.

$$
\mathrm{RILE}_{\mathrm{R}}=\left\lfloor\frac{200-(100-\mathrm{RILE})}{200}\right\rfloor * 10
$$

La segunda fuente de datos es el Base de Datos de Élites LatinoamericanasUniversidad de Salamanca (PELA-USAL), la cual obtiene información sobre la organización interna de los partidos políticos a partir de cuestionarios aplicados

\footnotetext{
Desde el 2014, MARPOR desarrolló una serie de nuevas subcategorías que permiten capturar de mejor manera el análisis de los programas de gobierno. Este cambio modifica la construcción del Índice RILE ya que se excluyen las subcategorías 202,2 y 605,2. Los países que se incluyen en la extensión del proyecto hacia América Latina tienen integrada estas nuevas subcategorías. Sin embargo, los datos previos al 2014, para los países que integran el proyecto original, no tienen la desagregación con estas nuevas subcategorías, siendo, el caso mexicano, uno de estos últimos casos. Para ver en detalle las nuevas subcategorías y la construcción del índice para los casos comprendidos en la extensión del Manifesto Project en América del Sur, revisar Ares y Volkens (2017).
} 
a diputados latinoamericanos (1993-2018). El proyecto permite cuantificar las respuestas de los diputados por partido y país. Para el caso específico de la investigación, la ideología de los partidos es observada a partir de la ubicación ideológica media otorgada por sus representantes en la escala que mide, del uno al diez, los posicionamientos de izquierda a derecha, siendo uno izquierda y diez derecha (Alcántara 2004: 88; Kitschelt et al. 2010).

\section{Dimensión Izquierda-Derecha (LR-PELA)}

Autoubicación ideológica de partido= izquierda 1, 2, 3, 4, 5, 6, 7, 8, 9, 10 derecha.

$$
\bar{x}_{j}=\frac{\left(x_{1}+x_{2}+\ldots . x_{n}\right)}{n}
$$

$\bar{x}_{j}$ : $\quad$ Media de las ubicaciones ideológicas asignadas al partido por cada miembro

$x_{n} \quad$ Ubicación ideológica del partido según cada miembro

$n: \quad$ Número miembros entrevistados

Con la intención de determinar la variable dependiente, se crea el índice de coherencia ideológico-programática (ICIP). El indicador calcula la diferencia entre el programa electoral y las posiciones ideológicas de los partidos políticos latinoamericanos según sus miembros (Ruiz y Otero 2013). El índice mide, en escala de cero a nueve, de más a menos coherencia, entendiendo con ello que los partidos que muestren valores cercanos a cero son partidos más coherentes. La unión de las dos perspectivas permite la comparación de ambas medidas y arroja información útil sobre la estructuración de la competencia política en torno a la ideología de los partidos.

Índice de coherencia ideológico-programática (ICIP):

Coherencia $0,1,2,3,4,5,6,7,8,9$ Incoherencia

$$
I C I P=\sqrt{\left(R I L E_{r}-P E L A_{i d p}\right)^{2}}
$$

PELA $_{\text {idp }}$ : Promedio del partido según la autoubicación de sus miembros en escala 1-10

RILE $_{\mathrm{r}}$ : Posiciones ideológicas del partido según el programa electoral en escala 1-10

** : $\quad$ Entre más alejado del 0, más incoherencia ideológica

Los casos que se analizan en la presente investigación se seleccionaron a partir de tres criterios: el primero es la selección según el método de la diferencia respecto a la existencia de gobiernos de coalición, el segundo criterio se da en función del número efectivo de partidos relativo a cada país y el tercer criterio, de naturaleza técnica, es la existencia de datos para cada partido y coalición en ambos proyectos a lo largo del tiempo, para, así, hacer posible su comparación. En este sentido, el proyecto PELA estudia a los parlamentarios de América Latina desde 1995, mientras que MARPOR comenzó su extensión hacia América 
Latina en las más recientes versiones del proyecto, ofreciendo datos desde 1989 para Chile y México (contemplado en la base original) desde 1945, lo que hace posible realizar esta comparación. De acuerdo con lo anterior, se observan los casos de Chile (1993-2017) y México (1994-2018), arrojando el análisis de veintidós partidos y cuatro coaliciones durante 1993-2018 (tabla 1). En ambos casos, uno, al ser presidencialismo de coalición y el otro, al promover coaliciones electorales, se analizan las distancias que puedan existir entre los parlamentarios, las propias coaliciones y las plataformas programáticas de los presidentes.

\section{Tabla 1. Los casos}

\begin{tabular}{|c|c|c|c|c|}
\hline \multirow[b]{2}{*}{ País } & \multirow[b]{2}{*}{ Partido/coalición } & MARPOR & \multicolumn{2}{|c|}{ PELA } \\
\hline & & Elecciones & Legislatura & $\begin{array}{c}\mathrm{N} \\
\text { militantes }\end{array}$ \\
\hline \multirow{9}{*}{ Chile } & Alianza (UDI; RN) & & \multirow{8}{*}{$\begin{array}{l}\text { 1993-1997; } \\
\text { 1997-2002; } \\
2002-2006 \\
2006-2010 \\
2010-2014 \\
2014-2018\end{array}$} & 278 \\
\hline & $\begin{array}{l}\text { Concertación por la Democracia / Nueva } \\
\text { Mayoría (PS; PDC; PRSD; PPD) }\end{array}$ & & & 294 \\
\hline & Partido Demócrata Cristiano (PDC) & & & 135 \\
\hline & Partido por la Democracia (PPD) & $\begin{array}{l}1993 ; 1999 ; \\
2002 ; 2005 ;\end{array}$ & & 80 \\
\hline & Partido Radical Socialdemócrata (PRSD) & $2009 ; 2013$ & & 28 \\
\hline & Partido Socialista (PS) & & & 51 \\
\hline & Renovación Nacional (RN) & & & 114 \\
\hline & Unión Demócrata Independiente (UDI) & & & 164 \\
\hline & 6 partidos; 2 coaliciones & 6 elecciones & 6 legislaturas & 1144 \\
\hline \multirow{11}{*}{ México } & $\begin{array}{l}\text { Alianza PRD: Alianza por México (Coalición } \\
\text { PRD, PT, PSN, PAS); Por el bien de todos (Coa- } \\
\text { lición PRD, MC, PT); Movimiento Progresista } \\
\text { (Coalición PRD, PT, MC) }\end{array}$ & & \multirow{10}{*}{$\begin{array}{l}\text { 1994-1994; } \\
\text { 1997-2000; } \\
2000-2003 ; \\
2003-2006 \\
2006-2009 ; \\
2009-2012 ; \\
2012-2015 ; \\
2015-2018\end{array}$} & 66 \\
\hline & $\begin{array}{l}\text { Alianza PRI-PVEM: Alianza por México, } \\
\text { Primero México, Compromiso por México }\end{array}$ & & & 117 \\
\hline & Salvemos México (Coalición PT, Mc) & & & 5 \\
\hline & $\begin{array}{l}\text { Partido Convergencia/Movimiento Ciuda- } \\
\text { dano (MC) }\end{array}$ & $\begin{array}{l}\text { 1994;1997; } \\
\text { 2000; 2003; } \\
\text { 2006; 2009; }\end{array}$ & & 4 \\
\hline & Partido Acción Nacional (PAN) & 2012 & & 248 \\
\hline & Partido Nueva Alianza (PANAL) & & & 4 \\
\hline & Partido de la Revolución Democrática (PRD) & & & 148 \\
\hline & Partido Revolucionario Institucional (PRI) & & & 349 \\
\hline & Partido del Trabajo (Рт) & & & 9 \\
\hline & Partido Verde Ecologista de México (PVEM) & & & 9 \\
\hline & 7 partidos; 3 coaliciones & 7 elecciones & 8 legislaturas & 959 \\
\hline $\mathrm{N}$ & 13 partidos; 5 coaliciones & 13 elecciones & 14 legislaturas & 2103 \\
\hline
\end{tabular}


El análisis de las coaliciones se observa en dos momentos. El primero refiere a la coherencia respecto a la media de las posiciones ideológicas, otorgadas por los miembros (LR PELA) de los partidos integrantes de cada coalición con relación a la postura evidenciada por la plataforma electoral (RILE MARPOR). El segundo refiere a las propias plataformas electorales. Específicamente, en el caso mexicano se presentan plataformas diferenciadas para formar coaliciones como por partido. En este caso, se toman las plataformas individuales cuando el análisis así lo requiere, y las plataformas en coalición cuando se analiza dicha condición. En el caso chileno solo se presentan plataformas de coalición / por candidato presidencial, por lo cual se toma la misma medida para analizar ambas condiciones. Los criterios establecidos para el análisis de estos dos sistemas de partidos asumen dos consecuencias teóricas. La primera responde a la observación de dos sistemas de partidos institucionalizados con dinámicas de competencia disímiles (Torcal 2015), entendiendo con ello que el sistema de partidos chileno responde teóricamente a dinámicas programáticas de competencia (Gamboa et al. 2013; López et al. 2013; Luna 2014; Ruiz y Miranda 2017) y el caso mexicano potencia el análisis de un sistema en proceso de polarización ideológica (Martínez-Hernández 2016; Martínez-Hernández y Martínez 2017). La segunda se asocia el interés analítico sobre el entendimiento de la ideología en sistemas presidenciales con gobiernos de coalición, como es el caso chileno, y sistemas con presidencialismos clásicos unipartidistas como el caso mexicano (Kitschelt et al. 2010; Torcal 2015). En este sentido, se esperarían dos supuestos: el primero es que los sistemas de partidos con niveles altos de institucionalización programática de competencia manifiesten mayores niveles de coherencia ideológico-programática. De acuerdo con lo anterior, dicha coherencia estaría condicionada a una historia institucional de colaboración partidista, como en el caso de los sistemas con diseño de gobiernos de coalición. El segundo es que los partidos políticos y coaliciones con posiciones ideológicas a la derecha de la escala manifiestan mayores niveles de coherencia, esto es debido a la divergencia en los procesos de la propia conformación de la ideología partidista (Freire 2015).

\section{ANÁLISIS Y DATOS}

El primer objetivo de la investigación es determinar el grado de coherencia de los partidos políticos y las coaliciones en México y Chile. Para ello se utilizan los tres indicadores mencionados en el apartado metodológico: el RILE-MARPOR, el LR-PELA y el índice de coherencia ideológica (ICIP). En términos generales, los resultados observados en el contexto mexicano y chileno se pueden resumir en dos elementos. El primero, es la propia distribución y diversidad de los partidos tanto en sus posiciones ideológicas (LR-PELA) como en el programa electoral (RILEMARPOR). En este ámbito, los partidos y coaliciones varían significativamente las magnitudes / distancia entre los dos indicadores ideológicos. La manifestación de estas diferencias se evidencia en tres patrones. El segundo tiene que ver con la distancia programática entre los partidos y las coaliciones o alianzas a las cuales 
se circunscriben. En este sentido, es posible observar a los partidos que más ceden programáticamente al interior de la coalición, esto es, los partidos que tienen mayor distancia entre el programa y su ubicación ideológica (miembros).

En el caso chileno, estos dos elementos de contraste pueden ser entendidos a partir de la propia estructura de su sistema de partidos. En estos términos, la política chilena durante su período democrático se ha caracterizado por la formación y competencia de dos grandes coaliciones donde se concentran los principales partidos políticos; las que, a pesar del tiempo, han variado poco. La división entre coaliciones está marcada por el posicionamiento de cada uno de los partidos respecto a la continuidad del régimen militar o al cambio democrático. Aquellos partidos que se mantienen proclives al apoyo del viejo régimen son la Unión Demócrata Independiente (UDI) y Renovación Nacional (RN), quienes conformaron la coalición de derecha actualmente denominada Chile Vamos y anteriormente llamada Alianza y Progreso, Alianza por Chile y Coalición por el Cambio.

Por otro lado, los partidos en su momento contrarios al régimen militar, se agruparon en una gran coalición para la democracia, conformada por el Partido Democracia Cristiana (PDC), el Partido Socialista (PS), el Partido por la Democracia (PPD) y el Partido Radical Socialdemócrata (PRSD) y, desde el 2013, el Partido Comunista (PC) (Picazo 2001: 245-247; Gamboa et al. 2013: 452-253).

En cuanto a los resultados a nivel individual, un primer patrón en el caso chileno muestra aquellos partidos y coaliciones más coherentes, en los que destacan los partidos de la coalición Nueva Mayoría (NM): el PS, PPD, PDC y el PRSD. En este sentido, se puede apreciar que la ubicación ideológica del PRSD es más cercana a la ubicación del programa electoral de la coalición, mientras que la ubicación ideológica del ps es la más alejada (gráficos 1 y 2). Lo anterior es relevante, dadas las características ideológicas de la propia coalición NM, en donde el partido ubicado más a la izquierda de la escala LR-PELA es quien menor coherencia manifiesta respecto al RILE-MARPOR. El segundo patrón permite observar aquellos partidos y coaliciones menos coherentes, como la Alianza de Chile, en donde RN y la UDI revelan los niveles más bajos de coherencia programática de los partidos y coaliciones analizados. La UDI muestra niveles de cercanía bajos en la mayoría de los casos. Existen dos momentos donde la coherencia es mayor, el primero entre el programa de 1993 y la primera legislatura analizada. El segundo es para la elección de 2005, cuando los dos principales partidos de la coalición deciden llevar un candidato propio a primera vuelta. En ambos casos, los programas de gobierno son los que tienen los posicionamientos más de derecha, y, a su vez, las posiciones de los diputados más cercanas hacia el centro, tal como se muestra en el gráfico 1. RN comparte el mismo programa de gobierno que la UDI, pero las posiciones de los parlamentarios están más cercanas que sus aliados dentro de la coalición, siendo el programa de 1993 donde existe una coherencia muy alta. 
Gráfico 1. Las diferencias entre los programas electorales y las posturas de los militantes de los partidos políticos y coaliciones en Chile (1993-2017)

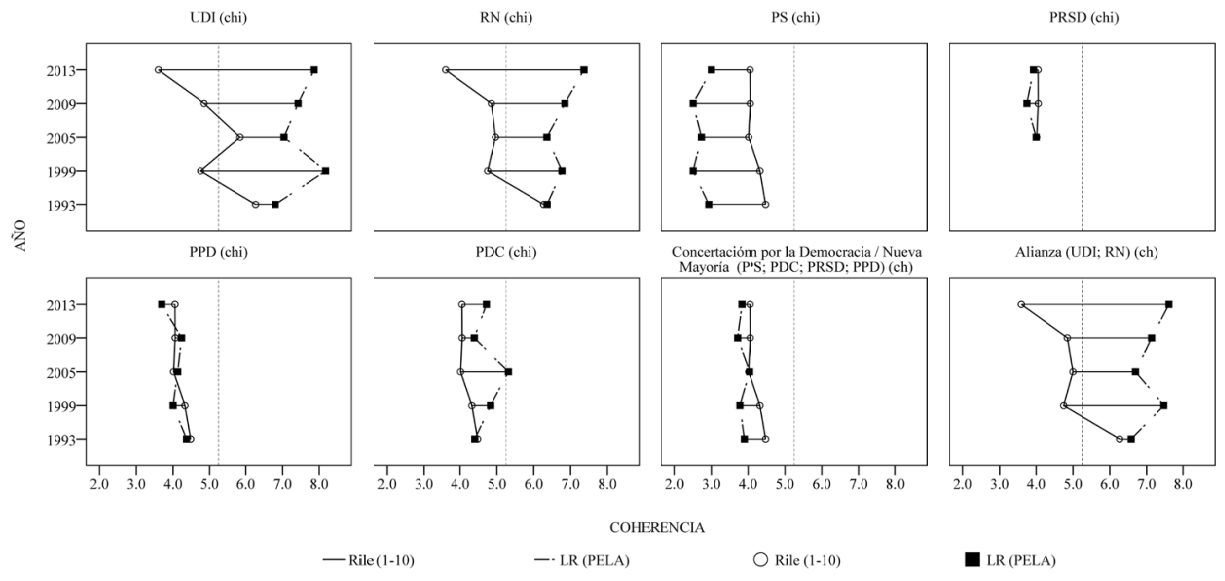

Fuente: elaboración propia con datos del MARPOR y PELA

Gráfico 2. La coherencia ideológico-programática (ICIP) de los partidos políticos en Chile (1993-2017)

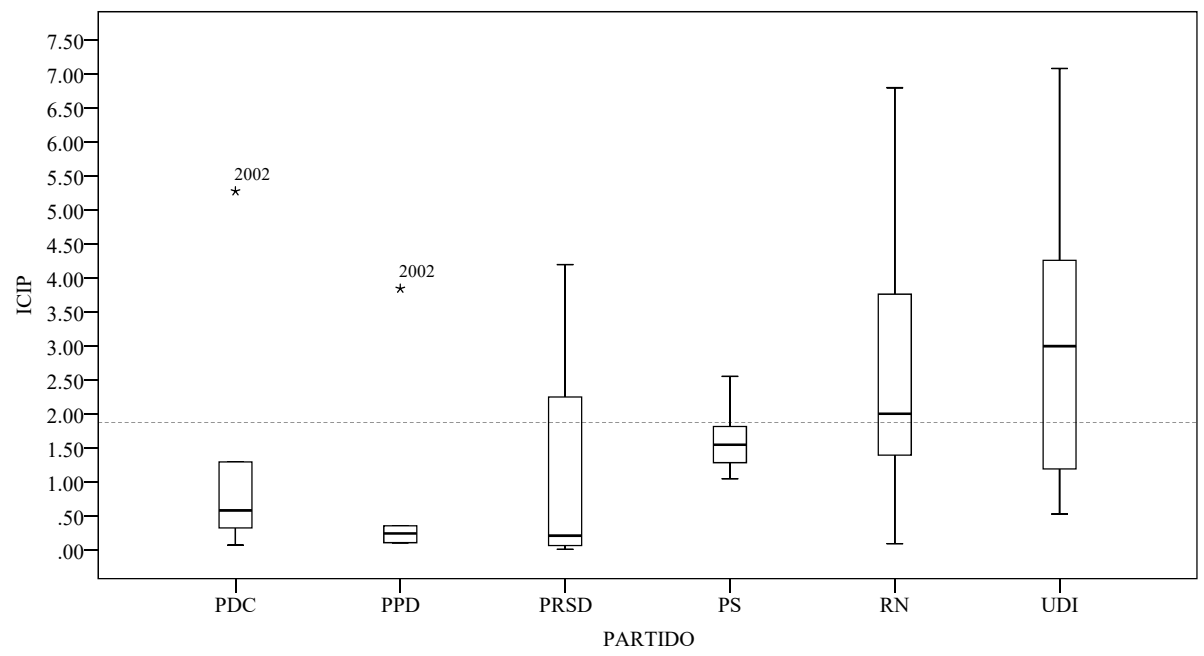

Fuente: elaboración propia con datos del MARPOR y PELA

En términos de coaliciones, como muestran los gráficos 3 y 4, tanto la UDI como RN muestran niveles bajos de coherencia. Las posiciones de los parlamentarios y el programa de gobierno de 1993 es el punto más cercano para ambos partidos, pero después de eso, los programas se desplazan hacia la izquierda (centro 
político), mientras que las posiciones de los diputados no lo hacen. El programa de gobierno de 2013 es sin dudas el principal desvío para ambos partidos de derecha. Un programa que, como se dijo, está más a la izquierda, incluso, que el de la coalición de centroizquierda (Ruiz y Miranda 2017). Sin embargo, las posiciones de los diputados continúan dentro de los rangos que presentan en las legislaturas anteriores (gráficos 3 y 4).

Gráfico 3. Las diferencias entre los programas electorales y las posturas de los militantes de la coalición de derecha en Chile (1993-2017)

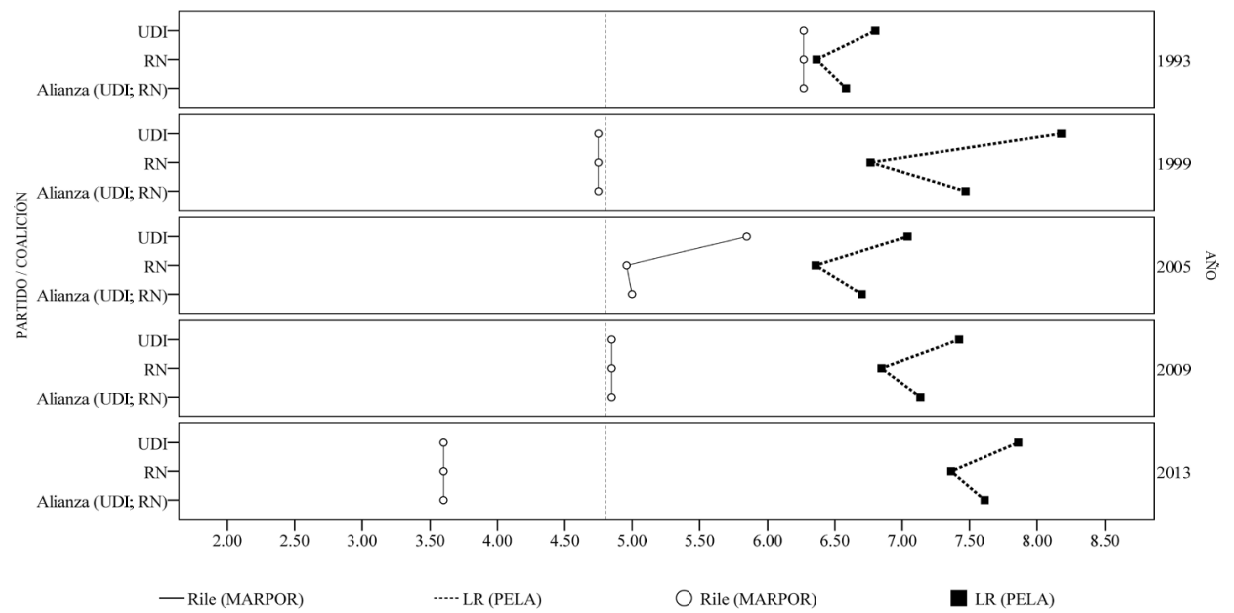

Fuente: elaboración propia con datos del MARPOR y PELA 
Gráfico 4. Las diferencias entre los programas electorales y las posturas de los militantes de la coalición de izquierda en Chile (1993-2017)

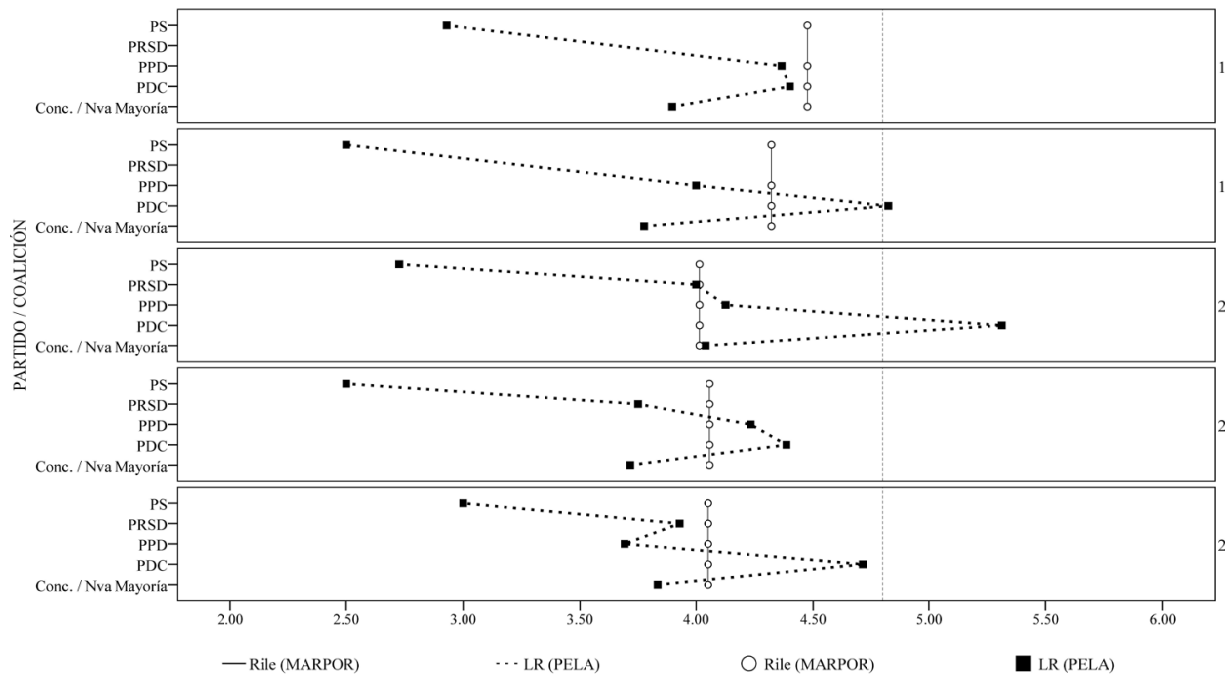

Fuente: elaboración propia con datos del MARPOR y PELA

La coalición de centroizquierda muestra resultados diferentes para los cuatro principales partidos analizados pero, en términos de coalición, presenta mejores niveles de coherencia que la coalición de derecha (Altman 2008: 47). El Ps y, en menor medida, la DC, muestran niveles de coherencia bajos, a pesar de que los candidatos presidenciales han salido de entre sus partidos. Por una parte, las posiciones de los diputados del ps se encuentran más a la izquierda que los programas de la coalición. Los momentos donde existe una coherencia cercana tienen en común que la candidata fue Michelle Bachelet, mientras que la coherencia más baja se da precisamente con el programa de otro candidato PS-PPD Ricardo Lagos.

Al contrario del PS, las posiciones de los diputados DC se encuentran más a la derecha que los programas (centro político) desde las elecciones de 1999, presentando los niveles de coherencia más bajos con el programa de las elecciones de 2005, con Michelle Bachelet. Como se observa en los gráficos 3 y 4, las elecciones de 1993, cuyo candidato pertenecía al Partido Demócrata Cristiano, la coherencia es muy cercana, algo similar ocurrió con el programa del mismo candidato en las elecciones de 2009, aunque, en esta oportunidad, las posiciones estarán más a la derecha. El PPD presenta niveles elevados de coherencia entre los programas de gobierno de la coalición y las posiciones de sus parlamentarios (gráfico 2). Existen pequeños desvíos con el programa de 1999 (candidato PS-PPD) y el 2013. De la misma manera, el PRSD presenta posiciones muy similares a los programas (gráfico 2), siendo los programas de 2005 y 2013 donde la coherencia es mayor, como se muestra en los gráficos 3 y 4. 
De esta manera, se puede observar a nivel de coalición que, a pesar de las diferencias existentes entre los partidos, los niveles de coherencia son mejores a la coalición de derecha. Las explicaciones de estos resultados se pueden encontrar en lo que Altman ha señalado respecto a que "la centroizquierda construye una coalición disciplinada y puede avanzar en su agenda porque logró definir objetivos políticos de manera compartida y, además, existió una predisposición de todos los partidos a la colaboración" (2008: 47). Dos de los cuatro partidos que la componen presentan posiciones muy cercanas a sus programas, lo que puede indicar un rol facilitador dentro de la configuración de las políticas que se desarrollen durante el gobierno. Los otros dos son los que presentan una distancia respecto a los programas, especialmente el PS, mientras que la DC mejorará su coherencia cuando el candidato pertenece a su partido. Los dos partidos que se posicionan en los extremos de la coalición son los que podrían manifestar un rol más contradicciones en las negociaciones durante la elaboración de políticas.

El caso mexicano, por su parte, muestra resultados similares al chileno para los tres partidos importantes del sistema. El Partido Revolucionario Institucional (PRI) es uno de los partidos que más cercanía tiene entre sus programas de gobierno y las posiciones de sus representantes. El programa del 2000 y la tercera legislatura analizada, presenta una coherencia ideológica muy alta, mientras que en los otros casos se observan pequeñas diferencias hacia la derecha de los programas (centro político). Por su parte, el Partido Acción Nacional (PAN) muestra una distancia mayor entre los programas y las posiciones de sus representantes, como se observa en el gráfico 5. El caso más cercano entre ambos se produce entre el programa de 1997 y la segunda legislatura, pero luego la diferencia va aumentando hasta las últimas elecciones, cuando las posiciones de vuelven más hacia la derecha de la escala ideológica, mientras que los programas se posicionan hacia el centro político (gráfico 5). El Partido de la Revolución Democrática (PRD) es el partido que mejores resultados de coherencia ideológica presenta entre los principales partidos (gráfico 6). En tres de los casos comparados (1994, 2000 y 2003), tanto programas como posiciones de los representantes tienen niveles de coherencia muy altos, mientras que los otros casos presentan una pequeña diferencia, como se observa en el gráfico 5. La mayoría de estas desviaciones se produce porque los programas se encuentran a la derecha (o hacia el centro político) de las posiciones, exceptuando el 2009, cuando las posiciones se ubicaron levemente más hacia el centro político (gráficos 5 y 6). El Partido Verde Ecologista de México (PVEM) y el Partido Nueva Alianza (PANAL) presentan dos observaciones (2009 y 2012) similares al PRI, donde es posible advertir una pequeña diferencia entre los programas y las posiciones de los representantes. Mientras los programas electorales se ubicaron en el centro político, las posiciones de los miembros partidistas se movieron a la centroderecha. En el gráfico 5 es posible observar que el programa electoral del PVEM para el 2009 tiene más cercanía con las posiciones de los representantes, pero para el 2012 la distancia aumenta, no tanto por un cambio entre los programas, como sí lo serán las preferencias de los diputados en la 
última legislatura. Para el PANAL ocurre algo similar pero, en este caso, será la elección de 2009 donde se presentaron más diferencias entre las posiciones de los representantes y del programa, mientras que las del 2012 manifestaron mayor cercanía. Con el Partido del Trabajo (PT) la situación es similar; de sus cuatro observaciones, existen dos momentos con mayor coherencia (1997 y 2009), mientras que la distancia es mayor en el primer (1994) y último caso analizado (2012). En todos ellos la desviación se produce por una variación en las posiciones de los diputados, siendo relativamente constante las posiciones de los programas electorales (gráfico 5). Finalmente, el Movimiento Ciudadano (MC), para el cual también existen dos observaciones. Es posible ver que las posiciones de los representantes y los programas son muy cercanas, por lo que presenta uno de los niveles más altos de coherencia dentro del sistema político mexicano, no obstante, es el partido con menos observaciones (gráfico 5 y 6).

Gráfico 5. Las diferencias entre los programas electorales y las posturas de los militantes de los partidos políticos y coaliciones en México (1994-2018)

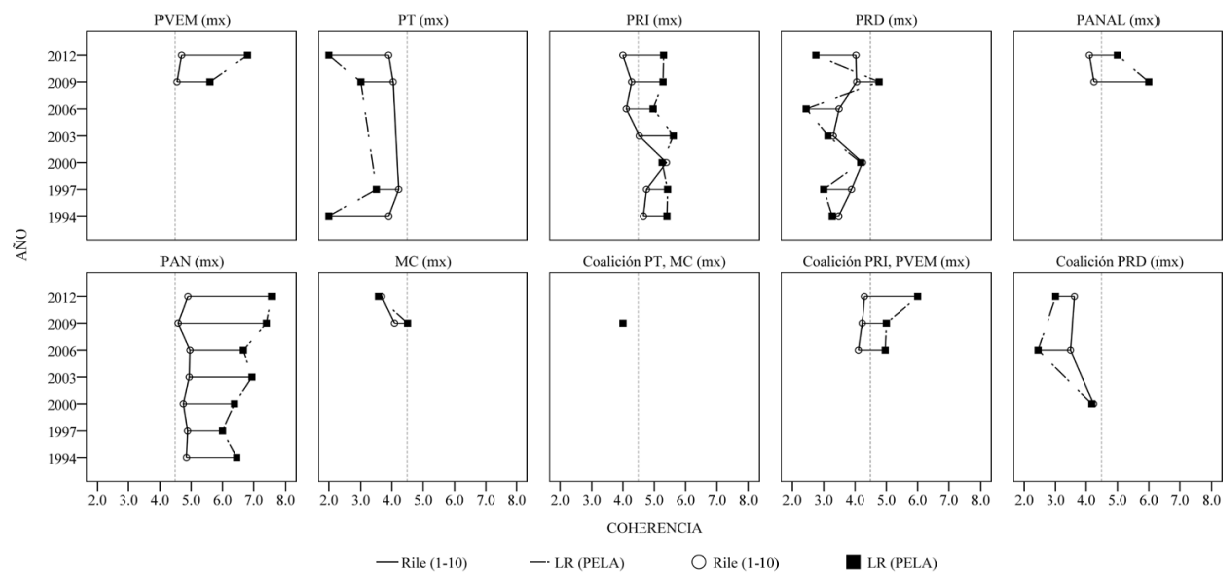

Fuente: elaboración propia con datos del MARPOR y PELA 
Gráfico 6. La coherencia ideológico programática (ICIP) de los partidos políticos en México (1994-2018)

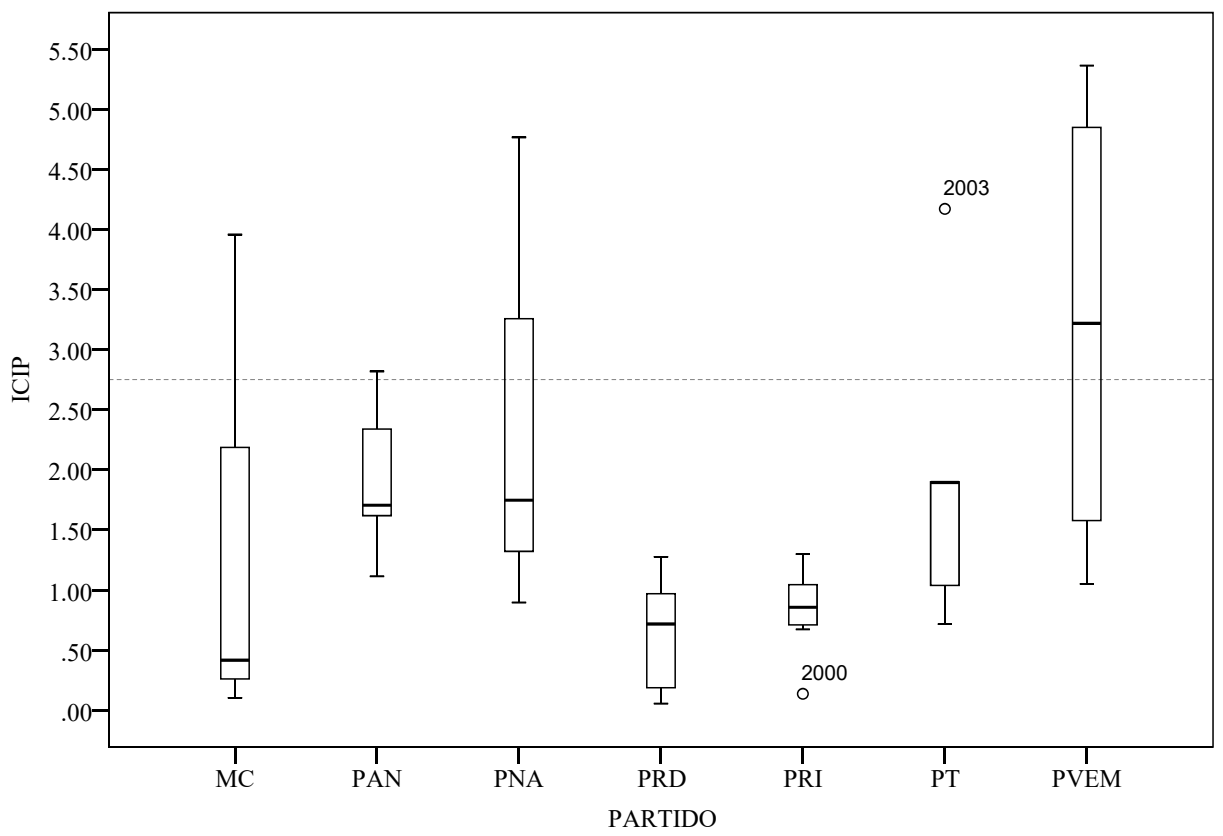

Fuente: elaboración propia con datos del MARPOR y PELA

Las coaliciones electorales en México tienen una característica en la conformación del sistema político durante las últimas dos décadas. Durante el período analizado, ellas han manifestado cambios relativos a su composición. Lo anterior tiene que ver principalmente con el propio sistema electoral, debido a que las coaliciones cambian dependiendo la elección que se trate, diferenciando entre elecciones legislativas y elecciones presidenciales. No obstante, se pueden apreciar dos grandes coaliciones electorales en el país, la coalición de centroderecha PRI-PVEM y la coalición de izquierda PRD-PT-MC. En concreto, los partidos PAN, PT y PVEM y la coalición Compromiso por México (PRI-PVEM) son los partidos y alianzas menos coherentes. El ejercicio evidenciado en los gráficos 5 y 6 muestra los niveles de coherencia programática (alta y baja) de los partidos y coaliciones mexicanos, indicando a los partidos y coaliciones con coherencia media como el Movimiento Progresista (PRD, PT, MC), así como el PRI, PRD y PANAL (gráficos 1 y 2).

Según la estructura de la coalición de centroderecha PRI-PVEM, la coherencia de esta coalición ha evidenciado cambios a lo largo de los procesos analizados. Las elecciones de 2006, 2009 y 2012 evidenciaron diferencias ideológicas, mostrando que las posiciones de los miembros de ambos partidos se movieron hacia la derecha de la escala, mientras su programa electoral mostró cierta continuidad 
hacia el centro ideológico. El PRI, por su parte, en los tres procesos analizados ha manifestado ubicaciones ideológicas más al centro de la escala, a pesar de que su programa electoral se mueve hacia la izquierda. El PVEM es el integrante de la coalición que manifestó ubicaciones ideológicas más hacia la derecha de la escala, destacando que los miembros partidistas de esta organización se posicionan más a la derecha que el programa electoral de la coalición. Las elecciones de 2012, que es cuando el PRI regresa a la presidencia de la República, manifiestan un cambio relativo en la ubicación de sus militantes, que considerablemente se mueven hacia la derecha de la escala, mientras el programa electoral se mantiene más al centro ideológico en comparación a las elecciones anteriores de 2009 y 2012. En esta coalición, claramente el PVEM es el partido que manifiesta posiciones menos coherentes respecto al programa electoral de la coalición (gráfico 7).

Gráfico 7. Las diferencias entre los programas electorales y las posturas de los militantes de las coaliciones de centro y centro derecha en México (1994-2018)

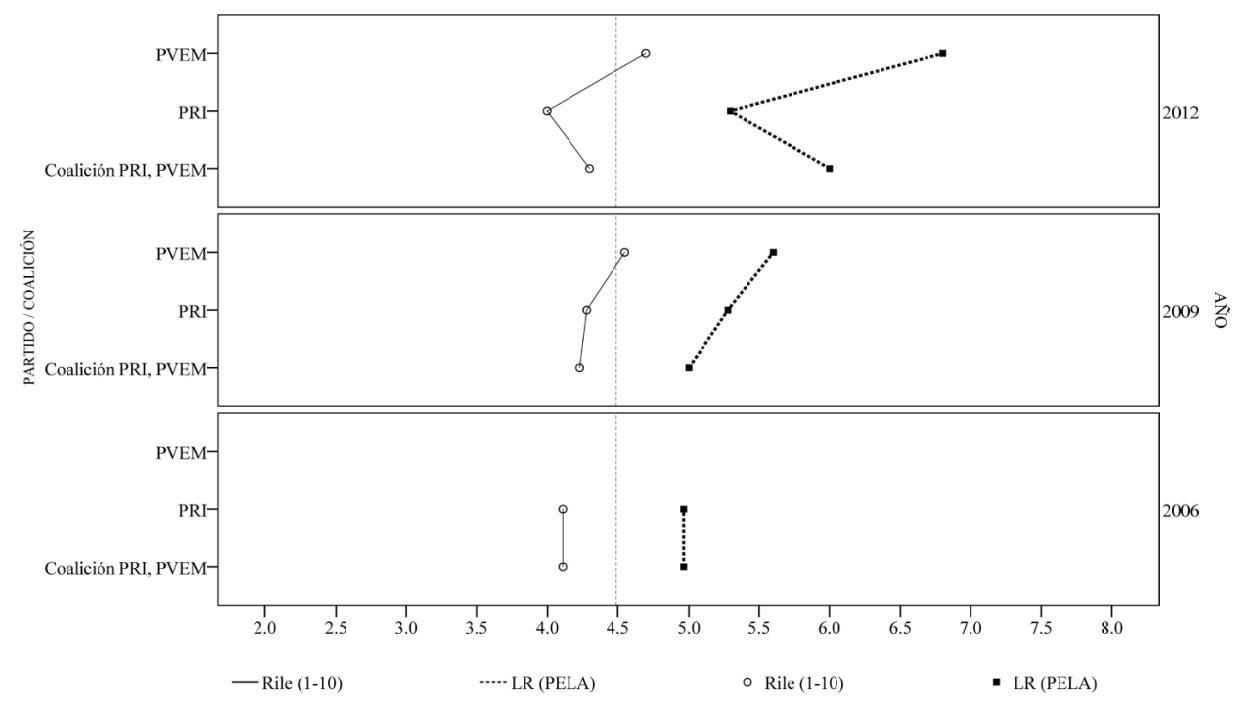

*Sin datos del pela para 2006 sobre el PVEM, integrante de la coalición de la coalición PRI-PVEM Fuente: elaboración propia con datos del MARPOR y PELA 
Por otro lado, según la coalición de centroizquierda integrada con regularidad por el PRD, PT y MC, es una alianza más coherente que la coalición PRI-PVEM. La evidencia muestra que la coalición ha mantenido posturas ideológicas de izquierda y centro desde las elecciones de 2006, 2009 y 2012 (gráfico 8). En este sentido, las ubicaciones ideológicas en las elecciones de 2006 y 2012, no solo manifiesta posiciones más a la izquierda de la escala, sino que son consistentes con niveles de coherencia inferiores a lo manifestado en las elecciones de 2009. En particular, las elecciones de 2012 manifiestan mayor coherencia que las elecciones de 2006. Estos resultados se deben a que, en estas dos elecciones, las posturas de los miembros de los partidos integrantes de esta coalición han manifestado posturas más a la izquierda que el programa electoral de la alianza electoral (gráfico 8).

Gráfico 8. Las diferencias entre los programas electorales y las posturas de los militantes de las coaliciones de izquierda y centro izquierda en México (1994-2018)

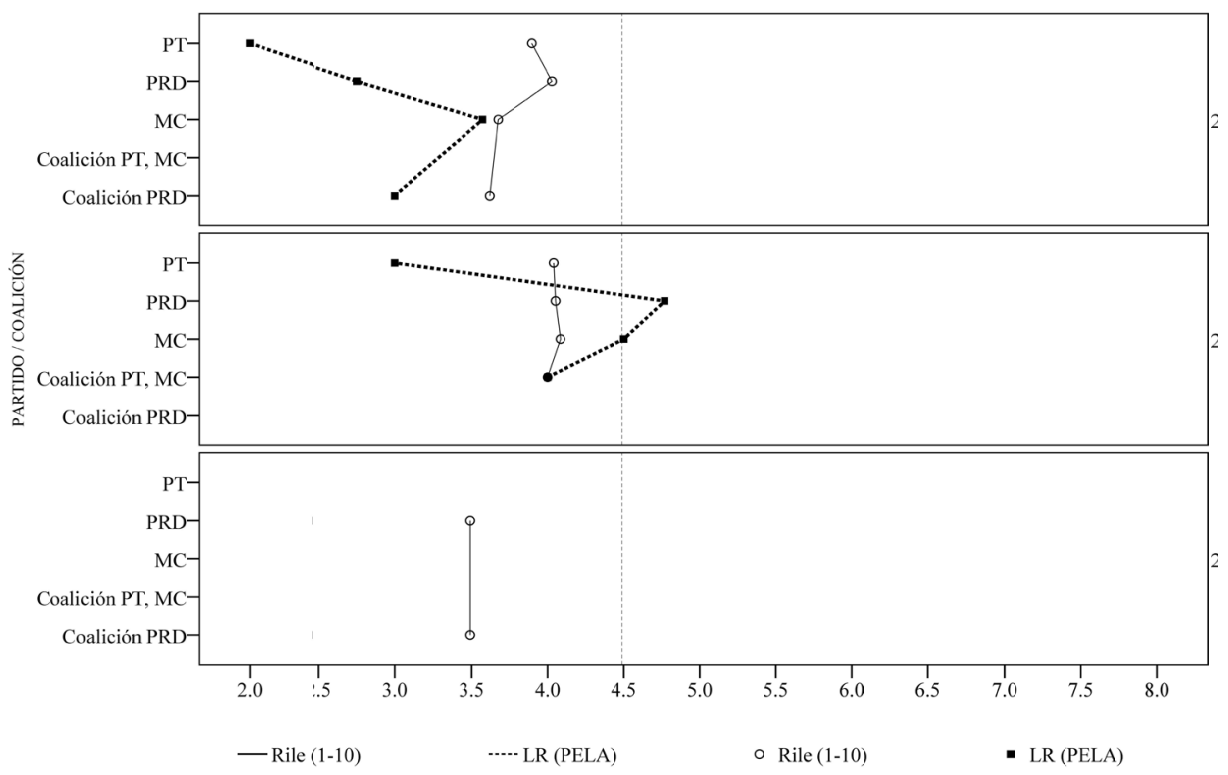

*Sin datos del PELA para 2006 sobre el PT y MC integrante de la coalición de la coalición PRD Fuente: elaboración propia con datos del MARPOR y PELA

En términos comparados y a nivel sistémico, Chile muestra niveles de coherencia más bajos que México (gráfico 9). Tal como se ha explicado, para el caso chileno, el programa de gobierno es una construcción común dentro de la coalición, donde es de esperar que existan diferencias entre los programas y las posiciones de los partidos, considerando, además, que cada uno de los partidos se ubica de manera diferenciada dentro del continuo izquierda-derecha. Sin embargo, la coalición de derecha presenta diferencias muy grandes entre el programa de 
gobierno y las posiciones de los representantes de RN y la UDI, cuyo caso más desviado entre ambas posiciones se da con el programa de gobierno de 2013 que se ubica muy a la izquierda, mientras que las posiciones de los diputados continúan en la derecha. En el caso de la coalición de centroizquierda, el partido que menos se ajusta a los programas de gobierno es el Ps, incluso en aquellos casos donde el candidato ha pertenecido a su propio partido. Los movimientos de la coherencia ideológica de ambos países hacen referencia a las formas en que los partidos políticos y sus coaliciones se relacionan. El caso mexicano, en particular, deja ver no solo niveles más altos de coherencia, también cambios en los procesos ideológicos, los cuales han sido más homogéneos que en el caso chileno.

Gráfico 9. Comparativo de la coherencia ideológico-programática entre los programas electorales y las posturas de los militantes en Chile y México (1993-2018)

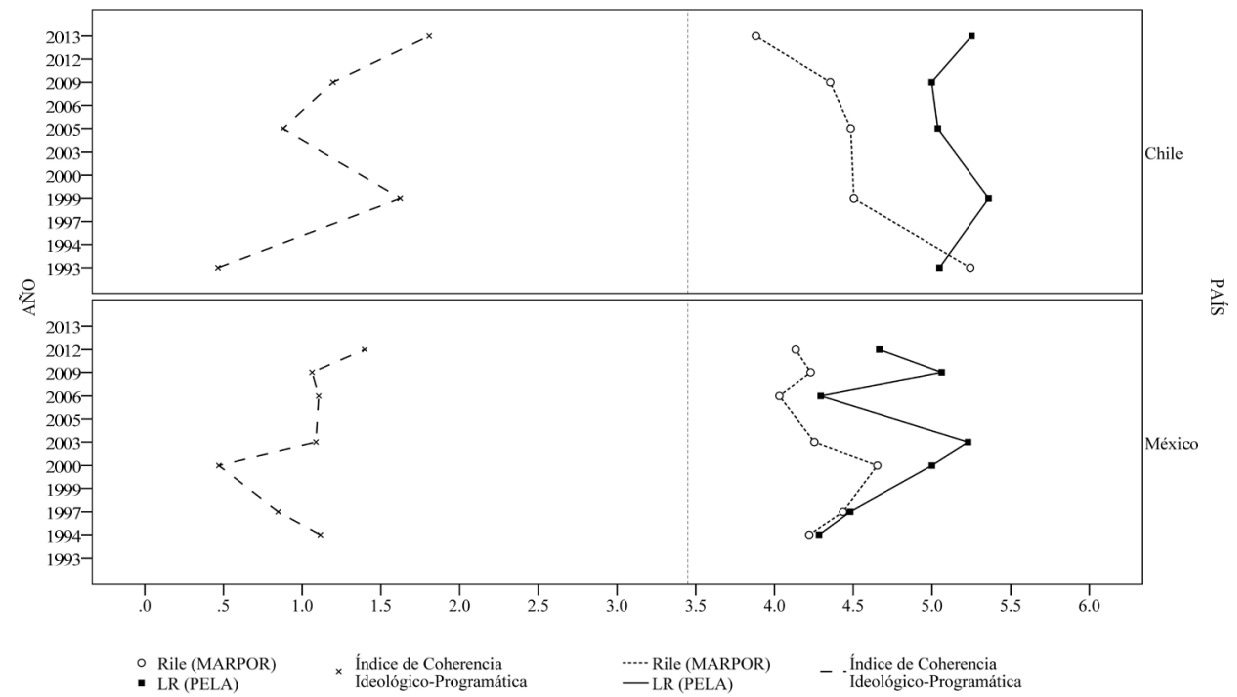

Fuente: elaboración propia con datos del MARPOR y PELA

Para entender lo anterior se pueden establecer dos aspectos relevantes en torno a la coherencia ideológico-programática de los partidos y coaliciones en América Latina, claramente centrados en los dos sistemas antes desarrollados. En primera instancia, la construcción del indicador de coherencia ideológica tiene que ver con la unión de las dos perspectivas RILE-MARPOR y LR-PELA, no obstante, la posición ideológica de los miembros de los partidos (LR-PELA) tiene un mayor peso en la medición que la medida proporcionada por los programas electorales. De acuerdo con ello, el segundo aspecto manifiesta que los partidos y coaliciones que mantienen posiciones a la derecha de la escala ideológica son menos coherentes programáticamente, como lo evidencia en mayor medida la 
Alianza (UDI y RN) chilena y el PAN mexicano. En este sentido, la coherencia es mayor con las posiciones ideológicas a la izquierda de la escala LR-PELA, como el mc de México o la coalición Nueva Mayoría de Chile. Lo anterior manifiesta que los partidos y coaliciones de derecha tienden a manifestar en sus programas electorales posturas ideológicas menos extremas que los propios miembros de estas organizaciones. Por ello, los cambios en los niveles de coherencia ideológica tienen relación con la percepción de los miembros en torno al proyecto ideológico de los partidos. Las posibles causas de los resultados anteriores hacen referencia a las propias estructuras de organización interna de los partidos. Por un lado, se observa la falta de penetración del ideal ideológicoprogramático en las bases partidistas y, por el otro, la relativa moderación en los programas electorales durante las elecciones. A pesar de que los partidos circunscriben elementos programáticos relativamente distinguibles respecto a las posiciones ideológicas, estos dos elementos — falta de institucionalización y moderación del ideal programático- suponen claras muestras de procesos de restructuración ideológica en los procesos de competencia electoral, lo cual no tiende a permear en las posiciones de los miembros partidistas ajenos a las plataformas electorales.

\section{VII.CONCLUSIONES}

La investigación se estructuró en torno a la observación de la coherencia ideológico-programática de los partidos políticos y coaliciones en dos contextos latinoamericanos. La principal aportación evidenciada por el análisis desarrollado es la capacidad de comparar dos perspectivas teóricas sobre estructuración de la ideología en América Latina. En este sentido, la investigación permitió la definición de estrategias metodológicas constreñidas en tres elementos. El primero es la creación del índice de coherencia ideológicoprogramática, el cual permitió determinar las diferencias entre los programas electorales y las posiciones ideológicas de los partidos según sus miembros. De acuerdo con ello, la evidencia apunta a que los partidos y coaliciones ubicados a la derecha de la escala son menos coherentes que los partidos y coaliciones de izquierda. El segundo elemento es precisamente la posibilidad de creación de mecanismos que permiten desarrollar análisis con fuentes de datos y posiciones teóricas distintas, que, en sí mismas, promueven estudios más robustos. La tercera es la concreción empírica del estudio de la ideología en contextos como el latinoamericano, saltando a la vista las divergencias y la gran diversidad programática de la región.

Esta investigación es una primera aproximación para el estudio de la coherencia ideológica y programática de los partidos políticos, y encuentra vínculos con los estudios conexos a tres grandes realidades teóricas. La primera se relaciona con los tipos de organización partidista en América Latina, destacando los estudios de la coherencia ideológica (interna), así como la verificación de las tipologías de partidos con una pretensión más programática en contraposición 
con los partidos pragmáticos-electorales como los catch all o cartel. La segunda refiere a los estudios de congruencia programática (partidos-ciudadanos), lo cual tiene que ver con la orientación programática de los partidos y la propia estructuración de la competencia. El tercero es precisamente las diferencias ideológicas en los gobiernos de coalición y sus implicaciones en los sistemas presidenciales, lo cual tiene que ver con los estudios relativos a los cambios y giros ideológicos en América Latina y sus implicaciones en la relación entre los poderes del Estado. Estos tres grandes temas abren una gama de líneas de investigación en torno a la representación política y el estado que guardan las democracias de la tercera ola de democratización y sus efectos en sus procesos de consolidación.

\section{REFERENCIAS}

Alcántara Sáez, Manuel. 2004. ¿Instituciones o máquinas ideológicas? Origen, programa y organización de los partidos politicos latinoamericanos. Barcelona: Institut de Ciencies Politiques i Socials-ICPS.

Alcántara Sáez, Manuel (dir.). 2018. Proyecto Elites Latinoamericanas de la Universidad de Salamanca (PELA-USAL) (1995-2018). Recuperado el 10 de abril de 2018 de https://oir.org. es/pela/

Alcántara Sáez, Manuel y Lina María Rincón Cabezas. 2013. “Estrategias electorales y funcionamiento interno de los partidos: selección de candidatos y programas electorales". En Selección de candidatos y elaboración de programas en los partidos políticos Latinoamericanos, editado por Manuel Alcántara Sáez y Lina María Cabezas Rincón. Ciudad de México: Tirant lo Blanch, 19-43.

Alonso Sáenz de Oger, Sonia, Andrea Volkens y Braulio Gómez Fortes. 2012. Análisis de contenido de textos políticos. Un enfoque cuantitativo. Madrid: Centro de Investigaciones Sociológicas (CIS).

Altman, David. 2008. "Régimen de gobierno y sistema de partidos en Chile". En Reforma de los partidos políticos en Chile, editado por Arturo Fontaine, Cristián Larroulet, Jorge Navarrete e Ignacio Walker. Santiago: Programa de las Naciones Unidas para el Desarrollo (PNUD), Centro de Estudios Públicos (CEP), Instituto Libertad y Desarrollo, Proyectamérica y la Corporación de Estudios para Latinoamérica (CIEPLAN), 41-74.

Ares, Cristina y Andrea Volkens. 2017. “¿Por qué y cómo se está extendiendo el Manifesto Project a América Latina?". Revista Española de Ciencia Política 43: 115-135.

Ares , Castro-Conde Cristina y Nicolás R. Miranda Olivares. 2017. “Aproximación al estudio del conflicto izquierda-derecha en Argentina, Brasil y Chile con datos del Manifesto Project". Trabajo preparado para su presentación en el Noveno Congreso Latinoamericano de Ciencia Política (ALACIP), Montevideo.

Avendaño, Octavio. 2008. “De la autonomía del mandato a la rendición de cuentas. Un alcance conceptual a los mecanismos de representación democrática". Revista de Sociología 22: 93-116.

Budge, Ian and Richard I. Hofferbert. 1990. "Mandates and Policy Outputs: U.S. Party Platforms and Federal Expenditures". American Political Science Review 84 (1): 111-131.

Budge, Ian, Hans-Dieter Klingemann, Andrea Volkens, Judith Bara and Eric Tanenbaum. 2001. Mapping Policy Preferences. Estimates for Parties, Electors, and Governments 19451998. Oxford: Oxford University Press.

Budge, Ian. 2006. "Identifying Dimensions and Locating Parties: Methodological and Conceptual Problems". In Handbook of Party Politics, eds. by Richard S. Katz and William Crotty. London: SAGE Publications, 422-433. 
Budge, Ian. 2013. “The Standard Right-Left Scale". Manuscrito no publicado. Universidad de Essex, Reino Unido.

Coppedge, Michael J. 1997. "A Classification of Latin American Political Parties". Working Paper (24). Notre Dame: Kellogg Institute For International Studies.

Coppedge, Michael. 1998. "The Dynamic Diversity of Latin American Party Systems". Party Politics 4 (4): 547-568.

D'Alessandro, Martín. 2013. "Las plataformas electorales en la Argentina moderna". América Latina Hoy 65: 107-139.

Dalton, Russell J. 1985. "Political Parties and Political Representation: Party Supporters and Party Elites in Nine Nations". Comparative Political Studies 18 (3): 267-299.

Franzmann, Simon and André Kaiser. 2006. "Locating Political Parties in Policy Space: A Reanalysis of Party Manifesto Data". Party Politics 12 (2): 163-188.

Freidenberg, Flavia, Fátima García Díez e Iván Llamazares. 2006. “Instituciones políticas y cohesión ideológica. Un análisis multinivel de la heterogeneidad ideológica en los partidos latinoamericanos". En Políticos y política en América Latina, editado por Manuel Alcántara Sáez. Madrid: Fundación Carolina y Siglo xxI editores, 255-280.

Freire, André. 2015. "Left-Right Ideology as a Dimension of Identification and of Competition". Journal of Political Ideologies 20 (1): 43-68.

Gamboa, Ricardo, Miguel Ángel López y Jaime Baeza. 2013. “La evolución programática de los partidos chilenos 1970-2009: De la polarización al consenso". Revista de Ciencia Política 33 (2): 443-467.

González Ferrer, Luis Eduardo y Rosario Queirolo Velasco. 2013. "Izquierda y derecha: formas de definirlas, el caso latinoamericano y sus implicaciones". América Latina Hoy 65: 79105.

Joignant, Alfredo, Mauricio Morales and Claudio Fuentes, eds. 2017. Malaise in Representation in Latin American Countries: Chile, Argentina, and Uruguay. New York: Palgrave Macmillan.

Kitschelt, Herbert, Kirk A. Hawkins, Juan Pablo Luna, Guillermo Rosas and Elizabeth J. Zechmeister. 2010. Latin American Party Systems. Cambridge: Cambridge University Press.

Laver, M. J. and Ian Budge, eds. 1992. Party Policy and Government Coalitions. New York: St. Martin's Press.

López-Varas, Miguel Ángel, Nicolás A. Miranda Olivares y Pablo A. Valenzuela-Gutiérrez. 2013. "Estimando el espacio político del Cono Sur y Brasil: las elecciones presidenciales en el eje izquierda-derecha". POstData: Revista de Reflexión y Análisis Político 18 (2): 403-442.

Luna, Juan Pablo and Elizabeth J. Zechmeister. 2005. "Political Representation in Latin America: A Study of Elite-Mass Congruence in Nine Countries". Comparative Political Studies 38 (4): 388-416.

Luna, Juan Pablo. 2014. Segmented Representation. Political Party Strategies in Unequal Democracies. New York: Oxford University Press.

Mainwaring, Scott y Mariano Torcal. 2005. “La institucionalización de los sistemas de partidos y la teoría del sistema partidista después de la tercera ola democratizadora". América Latina Hoy 41: 141-173.

Mainwaring, Scott y Timothy R. Scully. 1995. “La institucionalización de los sistemas de partidos en América Latina". Revista de Ciencia Política 17 (1-2): 63-101.

Manin, Bernard, Adam Przeworski y Susan C. Stokes. 2002. "Elecciones y representación". Zona Abierta 100-101: 19-50.

Manin, Bernard. 1998. Los principios del gobierno representativo. Traduc. Fernando Vallespín Oña. Madrid: Alianza Editorial.

Martínez-Hernández Aldo Adrián. y Daniela I. Martínez Rosales. 2017. “La ideología de los partidos políticos en México: la estructura de la competencia y la dimensión izquierda-derecha (1946-2012)". Revista Latinoamericana de Política Comparada 13: 31-55.

Martínez-Hernández, Aldo Adrián. 2016. "La ideología de los partidos políticos en América Latina: una explicación programática". En Política y democracia: anversos y reversos, edi- 
tado por Manuel Alcántara Sáez, Martín Cuesta y Mario Serrafero. Buenos Aires: L\&C Editorial; Salamanca: Flacso-España, 95-128.

McDonald, Michael D. and Ian Budge. 2005. Elections, Parties, Democracy: Conferring the Median Mandate. New York: Oxford University Press.

Miller, Warren. E. and Donald E. Stokes. 1963. "Constituency Influence in Congress". American Political Science Review 57 (1): 45-56.

Morales Quiroga, Mauricio. 2014. “Congruencia programática entre partidos y votantes en Chile". Perfiles Latinoamericanos 22 (44): 59-90.

Otero Felipe, Patricia y Juan Antonio Rodríguez Zepeda. 2014. “Vínculos ideológicos y éxito electoral en América Latina". Política y gobierno 21 (1): 159-200.

Otero Felipe, Patricia y Juan Antonio Rodríguez Zepeda. 2015. "El papel de la ideología en los partidos latinoamericanos". Revista Latinoamericana de Política Comparada 9: 71-95.

Picazo Verdejo, Inés. 2001. "Chile". En Partidos políticos de América Latina. Cono Sur, editado por Manuel Alcántara Sáez y Flavia Freidenberg. Salamanca: Ediciones Universidad de Salamanca, 245-354.

Pitkin, Hanna F. 2013. El concepto de representación. Traduc. Ricardo Montoro Romero. Madrid: Centro de Estudios Políticos y Constitucionales.

Ruiz Rodríguez, Leticia M. y Mercedes García Montero. 2003. “Coherencia partidista en las élites parlamentarias latinoamericanas". Revista Española de Ciencia Política (8): 71-102.

Ruiz Rodríguez, Leticia M. y Nicolás Miranda Olivares. 2017. "La evolución programática de las coaliciones en Chile". Revista Latinoamericana de Política Comparada 13: 57-78.

Ruiz Rodríguez, Leticia M. y Patricia Otero Felipe. 2013. Indicadores de partidos y sistemas de partidos. Madrid: Centro de Investigaciones Sociológicas-cis.

Ruiz Rodríguez, Leticia M. 2006. “Coherencia partidista: la estructuración interna de los partidos políticos en América Latina". Revista Española de Ciencia Política 14: 87-114.

Ruiz Rodríguez, Leticia M. 2007. La coherencia partidista en América Latina. Parlamentarios y partidos. Madrid: Centro de Estudios Políticos y Constitucionales.

Samuels, David J. and Matthew S. Shugart. 2006. "Presidents, Prime Ministers, Parties, and Mandate-Representation: A Global Test". Ponencia presentada en 102nd Annual Meeting of the American Political Science Association, Philadelphia.

Thomassen, Jacques and Hermann Schmitt. 1997. "Policy representation". European Journal of Political Research 32 (2): 165-184.

Thomassen, Jacques. 1991. "Empirical Research into Political Representation. A Critical Reappraisal". En Politische Klasse und politische Institutionen. Probleme und Perspektiven der Elitenforschung, editado por Hans-Dieter Klingemann, Richard Stöss and Bernhard Weßels. Schriften des Zentralinstituts für sozialwissenschaftliche Forschung der Freien Universität Berlin (ehemals Schriften des Instituts für politische Wissenschaft), vol 66. Wiesbaden: VS Verlag für Sozialwissenschaften, 259-274.

Thomassen, Jacques J.A. 1994. “Empirical Research into Political Representation: Failing Democracy or Failing Models?". En Elections at Home and Abroad. Essays in Honor of Warren E. Miller, editado por M. Kent Jennings and Thomas E. Mann. Ann Harbor: University of Michigan Press, 237-265.

Torcal, Mariano, coord. 2015. Sistemas de partidos en América Latina. Causas y consecuencias de su equilibrio inestable. Barcelona: Anthropos.

Volkens, Andrea. 2007. "Strengths and Weaknesses of Approaches to Measuring Policy Positions of Parties". Electoral Studies 26(1): 108-120.

Volkens, Andrea and Judith Bara. 2013. "Presidential Versus Parliamentary Representation: Extending the Manifesto Estimates to Latin America". En Mapping Policy Preferences from Texts: Statistical Solutions for Manifesto Analysts, editado por Andrea Volkens, Judith Bara, Ian Budge, Michael D. McDonald and Hans-Dieter Klingemann. Oxford: Oxford University Press, 277-298.

Volkens, Andrea, Judith Bara, Ian Budge, Michael D. McDonald and Hans-Dieter Klingemann. 2013. Mapping Policy Preferences from Texts. Statistical Solutions for Manifesto Analysts. Oxford: Oxford University Press. 
Volkens, Andrea, Pola Lehmann, Theres Matthieß, Nicolas Merz, Sven Regel \& Annika Werner. 2016. "The Manifesto Project Dataset - Codebook. Manifesto Project (MRG / CMP / MARPOR". Version 2016b (2016): The Manifesto Data Collection. Manifesto Project (MrG / CMP / MARPOR). Version 2016b. Berlin: Wissenschaftszentrum Berlin für Sozialforschung (WZB).

Recibido: 10 de abril de 2018

Aceptado: 4 de abril de 2019

Aldo Adrián Martínez-Hernández: Doctor y maestro en Ciencia Política por la Universidad de Salamanca (España). Posee también el grado de maestro en Asuntos Políticos y Políticas Públicas por El Colegio de San Luis AC, de México y es licenciado en Ciencias Políticas y Administración Pública por la Universidad Autónoma de Aguascalientes (México), donde actualmente ejerce como profesor-investigador asociado y es además investigador del Instituto de Iberoamérica (España). Miembro del Sistema Nacional de Investigadores (SNI) del Consejo Nacional de Ciencia y Tecnología (CONACYT), México. Correo electrónico: aldomaher@usal.es

Nicolás Miranda Olivares: Estudiante de doctorado en Ciencia Política por la Universidad de Salamanca (España), misma institución en la que ya obtuvo el grado de máster en Estudios Latinoamericanos. También es magíster en Ciencia Política por la Universidad de Chile. Actualmente es becario del programa "Doctorado en el extranjero, Beca Chile" de la Comisión Nacional de Investigación Científica y Tecnológica (CONICYT) del Estado de Chile. Correo electrónico: nicolas. miranda@usal.es 


\section{ANEXO}

Tabla 2. Descriptivos base de datos coherencia ideológica de los partidos políticos y coaliciones en Chile y México (1993-2018)

\begin{tabular}{|c|c|c|c|c|c|}
\hline Party / coal & & $\begin{array}{l}\text { Rile (MAR- } \\
\text { POR) }\end{array}$ & Lr (PELA) & $\begin{array}{l}\text { Índice de } \\
\text { coherencia } \\
\text { ideológica }\end{array}$ & $\begin{array}{l}\text { IDP (com- } \\
\text { puesto) }\end{array}$ \\
\hline \multirow{3}{*}{$\begin{array}{l}\text { Alianza (UDI; RN) } \\
\text { (Chi) }\end{array}$} & M & 4,892900 & 7,073670 & 2,996250 & 5,575540 \\
\hline & $\mathrm{N}$ & 5 & 6 & 6 & 6 \\
\hline & Sd. & 0,948186 & 0,412786 & 2,282840 & 1,066311 \\
\hline \multirow{3}{*}{ Coalición PRD (Mx) } & M & 3,782580 & 3,206670 & 0,575920 & 3,494630 \\
\hline & $\mathrm{N}$ & 3 & 3 & 3 & 3 \\
\hline & Sd. & 0,399768 & 0,890178 & 0,499570 & 0,643213 \\
\hline \multirow{3}{*}{$\begin{array}{l}\text { Coalición PRI, PVEM } \\
(\mathrm{Mx})\end{array}$} & $\mathrm{M}$ & 4,210680 & 5,321000 & 1,110320 & 4,765840 \\
\hline & $\mathrm{N}$ & 3 & 3 & 3 & 3 \\
\hline & Sd. & 0,097001 & 0,588322 & 0,514104 & 0,334201 \\
\hline \multirow{3}{*}{ Coalición PT, MC } & M & 3,999200 & 4,000000 & 0,000800 & 3,999600 \\
\hline & $\mathrm{N}$ & 1 & 1 & 1 & 1 \\
\hline & Sd. & 0,0 & 0,0 & 0,0 & 0,0 \\
\hline \multirow{4}{*}{$\begin{array}{l}\text { Concertación por la } \\
\text { Democracia / Nueva } \\
\text { Mayoría (PS; PDC; } \\
\text { PRSD; PPD) (Chi) }\end{array}$} & M & 4,183650 & 3,872330 & 0,946290 & 3,679350 \\
\hline & $\mathrm{N}$ & 5 & 6 & 6 & 6 \\
\hline & Sd. & 0,203685 & 0,121479 & 1,495530 & 0,836329 \\
\hline & M & 3,905650 & 4,035500 & 1,492820 & 3,297990 \\
\hline \multirow[t]{3}{*}{ MC (Mx) } & $\mathrm{N}$ & 3 & 2 & 3 & 3 \\
\hline & Sd. & 0,209482 & 0,656902 & 2,140920 & 1,190097 \\
\hline & M & 4,838090 & 6,775140 & 1,937050 & 5,806620 \\
\hline \multirow[t]{3}{*}{ PAN (Mx) } & $\mathrm{N}$ & 7 & 7 & 7 & 7 \\
\hline & Sd. & 0,130705 & 0,566964 & 0,613873 & 0,273964 \\
\hline & M & 4,37438 & 5,500000 & 2,470780 & 4,020530 \\
\hline \multirow[t]{3}{*}{ PANAL (Mx) } & $\mathrm{N}$ & 3 & 2 & 3 & 3 \\
\hline & Sd. & 0,348568 & 0,707107 & 2,034377 & 1,446126 \\
\hline & M & 4,183650 & 4,819670 & 1,358410 & 4,153020 \\
\hline \multirow[t]{3}{*}{ PDC (Chi) } & $\mathrm{N}$ & 5 & 6 & 6 & 6 \\
\hline & Sd. & 0,203685 & 0,407367 & 1,963802 & 0,757453 \\
\hline & M & 4,183650 & 4,043000 & 0,820240 & 3,764690 \\
\hline \multirow[t]{2}{*}{ PPD (Chi) } & $\mathrm{N}$ & 5 & 6 & 6 & 6 \\
\hline & Sd. & 0,203685 & 0,248583 & 1,486032 & 0,919297 \\
\hline
\end{tabular}




\begin{tabular}{|c|c|c|c|c|c|}
\hline Party / coal & & $\begin{array}{l}\text { Rile (MAR- } \\
\text { POR) }\end{array}$ & Lr (PELA) & $\begin{array}{l}\text { Índice de } \\
\text { coherencia } \\
\text { ideológica }\end{array}$ & $\begin{array}{l}\text { IDP (com- } \\
\text { puesto) }\end{array}$ \\
\hline \multirow{3}{*}{ PRD (Mx) } & M & 3,782480 & 3,364000 & 0,623510 & 3,573240 \\
\hline & $\mathrm{N}$ & 7 & 7 & 7 & 7 \\
\hline & Sd. & 0,355275 & 0,823649 & 0,481954 & 0,530969 \\
\hline \multirow{3}{*}{ PRI (Mx) } & $\mathrm{M}$ & 4,527240 & 5,317290 & 0,828990 & 4,922260 \\
\hline & $\mathrm{N}$ & 7 & 7 & 7 & 7 \\
\hline & Sd. & 0,469939 & 0,197784 & 0,371781 & 0,277553 \\
\hline \multirow{3}{*}{ PRSD (Chi) } & M & 4,040900 & 3,969750 & 1,160930 & 3,500210 \\
\hline & $\mathrm{N}$ & 3 & 4 & 4 & 4 \\
\hline & Sd. & 0,023264 & 0,186083 & 2,029656 & 0,934576 \\
\hline \multirow{3}{*}{ PS (Chi) } & M & 4,183650 & 2,702000 & 1,636380 & 3,094190 \\
\hline & $\mathrm{N}$ & 5 & 6 & 6 & 6 \\
\hline & Sd. & 0,203685 & 0,220854 & 0,521169 & 0,901617 \\
\hline \multirow{3}{*}{ PT (Mx) } & M & 4,044740 & 2,625000 & 1,944740 & 3,072370 \\
\hline & $\mathrm{N}$ & 5 & 4 & 5 & 5 \\
\hline & Sd. & 0,149231 & 0,750000 & 1,350215 & 0,674807 \\
\hline \multirow{3}{*}{ PVEM (Mx) } & M & 4,736080 & 6,200000 & 3,213950 & 3,918040 \\
\hline & $\mathrm{N}$ & 4 & 2 & 4 & 4 \\
\hline & Sd. & 0,444592 & 0,848528 & 1,982594 & 1,758385 \\
\hline \multirow{3}{*}{ RN (Chi) } & $\mathrm{M}$ & 4,884810 & 6,749330 & 2,678660 & 5,410000 \\
\hline & $\mathrm{N}$ & 5 & 6 & 6 & 6 \\
\hline & Sd. & 0,947216 & 0,372123 & 2,339925 & 1,023602 \\
\hline \multirow{3}{*}{ UDI (Chi) } & M & 5,0618200 & 7,397330 & 3,179150 & 5,807760 \\
\hline & $\mathrm{N}$ & 5 & 6 & 6 & 6 \\
\hline & Sd. & 1,0425710 & 0,530320 & 2,357119 & 1,149595 \\
\hline \multirow{3}{*}{ Total } & M & 4,3705600 & 4,947290 & 1,680820 & 4,323520 \\
\hline & $\mathrm{N}$ & 81 & 84 & 89 & 89 \\
\hline & Sd. & 0,6012760 & 1,643831 & 1,686359 & 1,257311 \\
\hline
\end{tabular}

1.Media (M); $2 \mathrm{~N}$ (casos); 3 Sd. (desviación estándar)

Fuente: elaboración propia con datos del MARPOR y PELA 